\title{
Exponential spline for the numerical solutions of linear Fredholm integro-differential equations
}

\author{
Taherh Tahernezhad ${ }^{1}$ and Reza Jalilian ${ }^{*}$
}

\author{
"Correspondence: \\ rezajalilian72@gmail.com; \\ r.jalilian@razi.ac.ir \\ 'Department of Mathematics, Razi \\ University, Kermanshah, Iran
}

\begin{abstract}
In this paper, we introduce a new scheme based on the exponential spline function for solving linear second-order Fredholm integro-differential equations. Our approach consists of reducing the problem to a set of linear equations. We prove the convergence analysis of the method applied to the solution of integro-differential equations. The method is described and illustrated with numerical examples. The results reveal that the method is accurate and easy to apply. Moreover, results are compared with the method in (J. Comput. Appl. Math. 290:633-640, 2015).
\end{abstract}

MSC: Primary 41A15; secondary 65RXX; 65D07; 34K05; 34K30

Keywords: Exponential spline; Boundary conditions; Integro-differential equation; Diagonally-dominant matrix; Convergence

\section{Introduction}

Integro-differential equations have gained a lot of interest in multitude of uses, specifically in sciences related to nature and engineering. Special usages of the integro-differential equations are visible in the mathematical modeling on spatio-temporal development of epidemics [44]. Generally, it is impossible to get an analytical answer for such equations. Because of that, various numerical methods have been devoted to finding the approximate solutions to such equations. The numerical solution of this type of integro-differential equations is discussed by a large number of authors. A few of these solutions are as follows: approximate solution that is obtained by using spline functions [1], Jacobi-spectral method for integro-delay differential equations with weakly singular kernels [25], polynomial spline functions that have free boundary condition for solving the first-order integrodifferential equations whose order of derivative is one [34], quartic trigonometric B-spline algorithm for numerical solution of the regularized long wave equation [15], an effective application of differential quadrature method based on modified cubic B-splines to numerical solutions of the $\mathrm{KdV}$ equation [3], and the exponential cubic b-spline collocation method for the Kuramoto-Sivashinsky equation in [18].

Recently, many authors have investigated the numerical methods for integral equations. These methods include a cubic spline approximation in $C^{2}$ to the solution of the Volterra

(c) The Author(s) 2020. This article is licensed under a Creative Commons Attribution 4.0 International License, which permits use, sharing, adaptation, distribution and reproduction in any medium or format, as long as you give appropriate credit to the original author(s) and the source, provide a link to the Creative Commons licence, and indicate if changes were made. The images or other third party material in this article are included in the article's Creative Commons licence, unless indicated otherwise in a credit line to the material. If material is not included in the article's Creative Commons licence and your intended use is not permitted by statutory regulation or exceeds the permitted use, you will need to obtain permission directly from the copyright holder. To view a copy of this licence, visit http://creativecommons.org/licenses/by/4.0/. 
integral equation of the second kind [33], quintic B-spline method [30], Bernstein operational matrix of derivative [4], hybrid of block pulse functions and normalized Bernstein polynomials [5], iterative method [49], sinc-collocation method [48], bivariate splines on nonuniform partitions [36], Jacobi operational matrices for solving delay or advanced integro-differential equations [40], the tau approximation for the Volterra-Hammerstein integral equations [21], b-spline collocation and cubature formulas [12] and [37], wavelet method [6], Walsh function method [35], Chebyshev finite difference method [13], differential transform method [7], Legendre polynomial method [39], an approximating solution, based on Lagrange interpolation and spline functions, to treat functional integral equations of Fredholm type and Volterra type [20], CAS wavelets method [22], an efficient matrix method based on Bell polynomials for solving nonlinear Fredholm-Volterra integral equations [32], collocation methods [10], Taylor polynomial methods [46], and Bernoulli matrix method [9]. Xuhao Li and Patricia J.Y. Wong in [26-29] have successfully applied non-polynomial spline to fractional diffusion problems. Besides, non-polynomial splines have also been applied to solve a system of second-order boundary value problems in the mid-knots of the mesh [14]. In addition, Sezer's method is discussed by Sezer et al. for approximating different types of integral and differential equations, especially Fredholm integro-differential equation [2]. Some papers have also developed numerical methods based on B-spline collocation method, for example, the extended B-spline collocation method for numerical solutions of Fisher equation in [17], numerical solutions of the Gardner equation by extended form of the cubic b-splines in [24], and in [23] generation of the trigonometric cubic b-spline collocation solutions for the Kuramoto-Sivashinsky (KS) equation.

For second-order impulsive integro-differential equations, periodic boundary value problems are discussed in [47]. Moreover, for second-order impulsive integro-differential equations, a class of three-point boundary value problems in Banach space have been developed in [19]. Yüzbaşi et al. in [50-56] used the non-polynomial functions to solve differential equations that have been based on non-polynomial functions set $\left\{1, e^{-t}, e^{-2 t}, \ldots\right\}$.

In this paper, based on the non-polynomial spline basis and quasilinearization method to solve the nonlinear Volterra integral equation [31], we want to use the non-polynomial spline functions to develop a numerical method for the solution of the Fredholm integrodifferential equation

$$
\left\{\begin{array}{l}
u^{\prime \prime}(x)+p(x) u^{\prime}(x)+q(x) u(x)=f(x)+\int_{a}^{b} k(t, x) u(t) d t \\
u(a)=\alpha, \quad u(b)=\beta, \quad t \in I:=[0,1]
\end{array}\right.
$$

where $p(x), q(x), k(t, x)$ are known functions and are considered sufficiently smooth, and also $u(x)$ is an unknown function to be determined. In [45] the existence of solutions has been discussed. In this paper, the basic ideas are developed to establish an algorithm that can be easily implemented and applied to second-order linear Volterra integro-differential equations. The aim of present work is to explore exponential spline interpolation with multiple parameters and devise a method to determine these parameters and also produce the minimum error. The main advantage of our algorithm is that it can be used directly without using assumption or transformation formulae.

The next sections of this paper are organized as follows. In Sect. 2, non-polynomial spline method to solve second-order boundary value problems of Fredholm integrodifferential equation is described. In Sect. 3, the convergence of the method is explained. 
The efficiency of the method by solving some examples and comparison of the numerical solutions with some other existing methods in [11] is shown in Sect. 4. Finally, a short conclusion is given.

\section{Exponential spline}

Proof of the existence and uniqueness of the non-polynomial interpolation function is presented in [42] and [43] (Sect. 2.3); in addition, the error analysis in non-polynomial interpolation function is proved in [41]. In [41] interpolation function has been presented as the form

$$
Q_{n}(x)=\sum_{i=0}^{n} c_{i} y_{i}(x)
$$

where $\left\{y_{0}(x), y_{1}(x), \ldots, y_{n}(x)\right\}$ are continuous functions which are real-valued and linearly independent on $[a, b]$; moreover $c_{0}, c_{1}, \ldots, c_{n}$ are coefficients which are determined by the interpolation conditions. The following form can be considered as a special item of (2):

$$
Q_{n}(x) \in \operatorname{span}\left\{e^{0 \lambda x}, e^{\lambda x}, e^{2 \lambda x}, \ldots,, e^{n \lambda x}\right\}
$$

Let $\Omega$ be a partition of the interval $[a, b]$, defined by the knots $x_{i}$, such that $\Omega: a=x_{0}<$ $x_{1}<\cdots<x_{n}=b$, with step size $h=\frac{b-a}{n}$. We denote the exponential spline function that interpolates the values $u_{0}, u_{1}, \ldots, u_{n}$ of the function of $u(x)$ by $S_{i}(x, \lambda)$ as follows:

$$
S_{\Omega}(x, \lambda)=a_{i} e^{\lambda\left(x-x_{i}\right)}+b_{i} e^{2 \lambda\left(x-x_{i}\right)}+c_{i} e^{3 \lambda\left(x-x_{i}\right)}+d_{i} e^{4 \lambda\left(x-x_{i}\right)} .
$$

The coefficients introduced in equations (3) are real and $\lambda$ is an arbitrary parameter. To derive expression for the coefficients in equations of (3) in terms of $u_{i}, u_{i+1}, M_{i}$ and $M_{i+1}$, we first denote

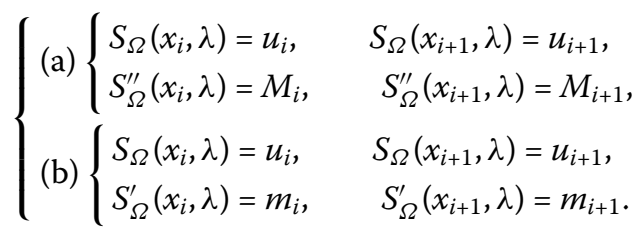

By using algebraic manipulation of (3) and (4)(a), we obtain the following relations:

$$
\begin{aligned}
\bar{a}_{i}= & e^{-\theta}\left(-e^{3 \theta}\left(-5+7 e^{\theta}\right) M_{i}+\left(7-5 e^{\theta}\right) M_{i+1}\right. \\
& \left.+4 \lambda^{2}\left(e^{3 \theta}\left(-20+7 e^{\theta}\right) u_{i}+\left(-7+20 e^{2 \theta}\right) u_{i+1}\right)\right), \\
\bar{b}_{i}= & e^{-2 \theta}\left(e^{3 \theta}\left(-8+7 e^{\theta}+7 e^{2 \theta}\right) M_{i}+\left(-7-7 e^{\theta}+8 e^{2 \theta}\right) M_{i+1}\right. \\
& \left.-\lambda^{2}\left(e^{3 \theta}\left(-128+7 e^{\theta}+7 e^{2 \theta}\right) u_{i}+\left(-7-7 e^{\theta}+128 e^{2 \theta}\right) u_{i+1}\right)\right), \\
\bar{c}_{i}= & e^{-2 \theta}\left(\left(e^{2 \theta}+e^{3 \theta}-4 e^{4 \theta}\right) M_{i}-\left(-4+e^{\theta}+e^{2 \theta}\right) M_{i+1}\right. \\
& \left.+4 \lambda^{2}\left(e^{2 \theta}\left(-4-4 e^{\theta}+e^{2 \theta}\right) u_{i}+\left(-1+4 e^{\theta}+4 e^{2 \theta}\right) u_{i+1}\right)\right), \\
\bar{d}_{i}= & e^{-2 \theta}\left(e^{2 \theta}\left(-3+5 e^{\theta}\right) M_{i}+\left(-3+5 e^{\theta}\right) M_{i+1}\right. \\
& \left.-\lambda^{2}\left(e^{2 \theta}\left(-27+5 e^{\theta}\right) u_{i}+\left(-5+27 e^{\theta}\right) u_{i+1}\right)\right),
\end{aligned}
$$




$$
\begin{aligned}
& \vartheta=3\left(-1+e^{\theta}\right)\left(7-18 e^{\theta}+7 e^{2 \theta}\right) \lambda^{2}, \\
& a_{i}=\frac{\bar{a}_{i}}{\vartheta}, \quad a_{i}=\frac{\bar{b}_{i}}{\vartheta}, \quad c_{i}=\frac{3 \bar{c}_{i}}{\vartheta}, \quad d_{i}=\frac{\bar{d}_{i}}{\vartheta} .
\end{aligned}
$$

To develop the consistency relations between the values of spline and its derivatives at knots, consider the following relation:

$$
\alpha M_{i-1}+2 \beta M_{i}+\alpha M_{i+1}=\frac{1}{h^{2}}\left(u_{i+1}-2 u_{i}+u_{i-1}\right),
$$

where

$$
\alpha=\frac{1}{(h \lambda)^{2}}\left(\frac{\lambda h}{\sinh (\lambda h)}-1\right), \quad \beta=\frac{1}{(h \lambda)^{2}}\left(1-\frac{\operatorname{coth}(\lambda h)}{\lambda}\right)
$$

and $\theta=h \lambda$.

Pay attention that exponential spline functions relation (5) will be identical with ordinary spline functions as $\theta \rightarrow 0$, which $(\alpha, \beta) \rightarrow\left(\frac{1}{6}, \frac{1}{3}\right)$. Moreover, assuming $\alpha=\frac{1}{12}, \beta=\frac{5}{12}$, we get the following relation:

$$
M_{i-1}+10 M_{i}+M_{i+1}=\frac{12}{h^{2}}\left(u_{i+1}-2 u_{i}+u_{i-1}\right), \quad i=1,2, \ldots, n-1 .
$$

By expanding (5) in Taylor series about $x_{i}$, we obtain the following local truncation error:

$$
\begin{aligned}
T_{i}= & (2 \alpha+2 \beta-1) h^{2} u_{i}^{\prime \prime}+\frac{1}{12}(12 \alpha-1) h^{4} u_{i}^{(4)}+\frac{1}{360}(30 \alpha-1) h^{6} u_{i}^{(6)} \\
& +\frac{(56 \alpha-1) h^{8} u^{(8)}}{20160}+O\left(h^{10}\right) .
\end{aligned}
$$

Similarly, by using (3) and (4)(b), we get

$$
\alpha m_{i-1}+2 \beta m_{i}+\alpha m_{i+1}=\frac{\alpha+\beta}{h}\left(u_{i+1}-u_{i-1}\right), \quad i=1,2, \ldots, n-1,
$$

and also by expanding (8) in Taylor series about $x_{i}$, we obtain the following local truncation error:

$$
T_{i}=\left(\frac{2 \alpha-\beta}{3(\alpha+\beta)}\right) h^{3} u_{i}^{(3)}+\left(\frac{4 \alpha-\beta}{60(\alpha+\beta)}\right) h^{5} u_{i}^{(5)}+\left(\frac{6 \alpha-\beta}{2520(\alpha+\beta)}\right) h^{7} u_{i}^{(7)}+O\left(h^{9}\right) .
$$

In the matrix notation, equations (5) and (8) have the following forms:

$$
W M=\bar{R} U, \quad Z m=S U,
$$

where $W, R, Z$, and $S$ are coefficient matrices in (5) and (8). We approximate $m_{0}=$ $\frac{-3 u_{0}+4 u_{1}-u_{2}}{2 h}$ and $m_{n}=\frac{3 u_{n-2}-4 u_{n-1}-u_{n}}{2 h}$, and also $M_{i}$ for $i=0, n$, by using second-order approximation. From (3) and (4) we have

$$
\begin{aligned}
S_{i}(x)= & \left(e ^ { \lambda ( x - x _ { i } ) } \left(5 e^{2 \theta} M_{i}-7 e^{3 \theta} M_{i}-5 M_{i+1}+7 e^{-\theta} M_{i+1}-80 e^{2 \theta} \lambda^{2} u_{i}+28 e^{3 \theta} \lambda^{2} u_{i}\right.\right. \\
& \left.\left.+80 \lambda^{2} u_{i+1}-28 e^{-\theta} \lambda^{2} u_{i+1}\right)\right)
\end{aligned}
$$




$$
\begin{aligned}
& /\left(3\left(-1+e^{\theta}\right)\left(7-18 e^{\theta}+7 e^{2 \theta}\right) \lambda^{2}\right) \\
& +\left(e ^ { 4 \lambda ( x - x _ { i } ) } \left(-3 M_{i}+5 e^{\theta} M_{i}-5 e^{-2 \theta} M_{i+1}+3 e^{-\theta} M_{i+1}+27 \lambda^{2} u_{i}-5 e^{\theta} \lambda^{2} u_{i}\right.\right. \\
& \left.\left.+5 e^{-2 \theta} \lambda^{2} u_{i+1}-27 e^{-\theta} \lambda^{2} u_{i+1}\right)\right) \\
& /\left(3\left(-1+e^{\theta}\right)\left(7-18 e^{\theta}+7 e^{2 \theta}\right) \lambda^{2}\right) \\
& +\left(e ^ { 2 \lambda ( x - x _ { i } ) } \left(-8 e^{\theta} M_{i}+7 e^{2 \theta} M_{i}+7 e^{3 \theta} M_{i}+8 M_{i+1}-7 e^{-2 \theta} M_{i+1}-7 e^{-\theta} M_{i+1}\right.\right. \\
& \left.\left.+128 \lambda^{2} e^{\theta} u_{i}-7 e^{2 \theta} \lambda^{2} u_{i}\right)\right) \\
& /\left(3\left(-1+e^{\theta}\right)\left(7-18 e^{\theta}+7 e^{2 \theta}\right) \lambda^{2}\right) \\
& +\frac{e^{2 \lambda\left(x-x_{i}\right)}\left(-7 \lambda^{2} e^{3 \theta} u_{i}-128 \lambda^{2} u_{i+1}+7 e^{-2 \theta} \lambda^{2} u_{i+1}+7 e^{-\theta} \lambda^{2} u_{i+1}\right)}{3\left(-1+e^{\theta}\right)\left(7-18 e^{\theta}+7 e^{2 \theta}\right) \lambda^{2}} \\
& +\left(e ^ { 3 \lambda ( x - x _ { i } ) } \left(M_{i}+e^{\theta} M_{i}-4 e^{2 \theta} M_{i}-M_{i+1}+4 e^{-2 \theta} M_{i+1}-e^{-\theta} M_{i+1}\right.\right. \\
& \left.\left.-16 \lambda^{2} u_{i}-16 e^{\theta} \lambda^{2} u_{i}\right)\right) \\
& /\left(\left(-1+e^{\theta}\right)\left(7-18 e^{\theta}+7 e^{2 \theta}\right) \lambda^{2}\right) \\
& +\frac{e^{3 \lambda\left(x-x_{i}\right)}\left(+4 \lambda^{2} e^{2 \theta} u_{i}+16 \lambda^{2} u_{i+1}+4 e^{-2 \theta} \lambda^{2} u_{i+1}+16 e^{-\theta} \lambda^{2} u_{i+1}\right)}{\left(-1+e^{\theta}\right)\left(7-18 e^{\theta}+7 e^{2 \theta}\right) \lambda^{2}}+O\left(h^{4}\right) .
\end{aligned}
$$

To discretize the integro-differential equation of (1) by using equation (4), we obtain

$$
\begin{aligned}
u_{i}^{\prime \prime}+ & p_{i} u_{i}^{\prime}+q_{i} u_{i} \\
= & f_{i}+\int_{a}^{b} k(t, x) u(t) d t \\
= & f_{i}+\sum_{j=0}^{n-1} \int_{t_{j}}^{t_{j+1}} k\left(t, x_{i}\right) u(t) d t \\
\approx & f_{i}+\sum_{j=0}^{n-1} \int_{t_{j}}^{t_{j+1}} k\left(t, x_{i}\right) S_{j}(t) d t, \\
= & f_{i}+\sum_{j=0}^{n-1} \frac{e^{2 \theta}\left(\left(5-7 e^{\theta}\right) M_{j}+\lambda^{2}\left(-80+28 e^{\theta}\right) u_{j}\right)}{3\left(-1+e^{\theta}\right)\left(7-18 e^{\theta}+7 e^{2 \theta}\right) \lambda^{2}} \int_{t_{j}}^{t_{j+1}} k\left(t, x_{i}\right) e^{\lambda\left(t-t_{j}\right)} d t \\
& +\sum_{j=0}^{n-1} \frac{\left(-5+7 e^{-\theta}\right) M_{j+1}+\lambda^{2}\left(80-28 e^{-\theta}\right) u_{j+1}}{3\left(-1+e^{\theta}\right)\left(7-18 e^{\theta}+7 e^{2 \theta}\right) \lambda^{2}} \int_{t_{j}}^{t_{j+1}} k\left(t, x_{i}\right) e^{\lambda\left(t-t_{j}\right)} d t \\
& +\sum_{j=0}^{n-1} \frac{e^{\theta}\left(\left(-8+7 e^{\theta}+7 e^{2 \theta}\right) M_{j}+\lambda^{2}\left(128-7 e^{\theta}-7 e^{2 \theta}\right) u_{j}\right)}{3\left(-1+e^{\theta}\right)\left(7-18 e^{\theta}+7 e^{2 \theta}\right) \lambda^{2}} \int_{t_{j}}^{t_{j+1}} k\left(t, x_{i}\right) e^{2 \lambda\left(t-t_{j}\right)} d t \\
& +\sum_{j=0}^{n-1} \frac{\left(8-7 e^{-\theta}-7 e^{-2 \theta}\right) M_{j+1}+\lambda^{2}\left(-128+7 e^{-\theta}+7 e^{-2 \theta}\right) u_{j+1}}{3\left(-1+e^{\theta}\right)\left(7-18 e^{\theta}+7 e^{2 \theta}\right) \lambda^{2}} \int_{t_{j}}^{t_{j+1}} k\left(t, x_{i}\right) e^{2 \lambda\left(t-t_{j}\right)} d t \\
& +\sum_{j=0}^{n-1} \frac{\left(1+e^{\theta}-4 e^{2 \theta}\right) M_{j}+\lambda^{2}\left(-16-16 e^{\theta}+4 e^{2 \theta}\right) u_{j}}{\left(-1+e^{\theta}\right)\left(7-18 e^{\theta}+7 e^{2 \theta}\right) \lambda^{2}} \int_{t_{j}}^{t_{j+1}} k\left(t, x_{i}\right) e^{3 \lambda\left(t-t_{j}\right)} d t \\
& +\sum_{j=0}^{n-1} \frac{\left(-1-e^{-\theta}+4 e^{-2 \theta}\right) M_{j+1}+\lambda^{2}\left(+16+16 e^{-\theta}-4 e^{-2 \theta}\right) u_{j+1}}{\left(-18 e^{\theta}+7 e^{2 \theta}\right) \lambda^{2}}
\end{aligned}
$$




$$
\begin{aligned}
& \times \int_{t_{j}}^{t_{j+1}} k\left(t, x_{i}\right) e^{3 \lambda\left(t-t_{j}\right)} d t \\
& +\sum_{j=0}^{n-1} \frac{\left(-3+5 e^{\theta}\right) M_{j}+\lambda^{2}\left(27-5 e^{\theta}\right) u_{j}}{3\left(-1+e^{\theta}\right)\left(7-18 e^{\theta}+7 e^{2 \theta}\right) \lambda^{2}} \int_{t_{j}}^{t_{j+1}} k\left(t, x_{i}\right) e^{4 \lambda\left(t-t_{j}\right)} d t \\
& +\sum_{j=0}^{n-1} \frac{e^{-\theta}\left(\left(3-5 e^{-\theta}\right) M_{j+1}+\lambda^{2}\left(-27+5 e^{-\theta}\right) u_{j+1}\right)}{3\left(-1+e^{\theta}\right)\left(7-18 e^{\theta}+7 e^{2 \theta}\right) \lambda^{2}} \int_{t_{j}}^{t_{j+1}} k\left(t, x_{i}\right) e^{4 \lambda\left(t-t_{j}\right)} d t
\end{aligned}
$$

We let

$$
\left\{\begin{array}{lll}
a(i, j)=\int_{t_{j}}^{t_{j+1}} k\left(t, x_{i}\right) e^{\lambda\left(t-t_{j}\right)} d t, & b(i, j+1)=\int_{t_{j}}^{t_{j+1}} k\left(t, x_{i}\right) e^{\lambda\left(t-t_{j}\right)} d t, \\
c(i, j)=\int_{t_{j}}^{t_{j+1}} k\left(t, x_{i}\right) e^{2 \lambda\left(t-t_{j}\right)} d t, & d(i, j+1)=\int_{t_{j}}^{t_{j+1}} k\left(t, x_{i}\right) e^{2 \lambda\left(t-t_{j}\right)} d t, \\
e(i, j)=\int_{t_{j}}^{t_{j+1}} k\left(t, x_{i}\right) e^{3 \lambda\left(t-t_{j}\right)} d t, & r(i, j+1)=\int_{t_{j}}^{t_{j+1}} k\left(t, x_{i}\right) e^{3 \lambda\left(t-t_{j}\right)} d t, \\
g(i, j)=\int_{t_{j}}^{t_{j+1}} k\left(t, x_{i}\right) e^{4 \lambda\left(t-t_{j}\right)} d t, & & h(i, j+1)=\int_{t_{j}}^{t_{j+1}} k\left(t, x_{i}\right) e^{4 \lambda\left(t-t_{j}\right)} d t,
\end{array}\right.
$$

and introduce the following relations:

$$
\left\{\begin{array}{llll}
a(i, n)=0, & b(i, 0)=0, & c(i, n)=0, & d(i, 0)=0, \\
e(i, n)=0, & r(i, 0)=0, & g(i, n)=0, & h(i, 0)=0 .
\end{array}\right.
$$

We can write the defined notations in the form of the matrix as follows: $A=\left(a_{i, j}\right), B=$ $\left(b_{i, j}\right), C=\left(c_{i, j}\right), D=\left(d_{i, j}\right), \bar{E}=\left(e_{i, j}\right), R=\left(r_{i, j}\right), G=\left(g_{i, j}\right), H=\left(h_{i, j}\right), Q=\left(q_{i, j}\right), P=\left(p_{i, j}\right)$ also if suppose $M \approx \hat{M}=\left(\widehat{M}_{0}, \widehat{M}_{1}, \ldots, \widehat{M}_{n-1}, \widehat{M}_{n}\right)^{T}, U \approx \hat{U}=\left(\widehat{u}_{0}, \widehat{u}_{1}, \ldots, \widehat{u}_{n-1}, \widehat{u}_{n}\right)^{T}, m \approx \hat{m}=$ $\left(\widehat{m}_{0}, \widehat{m}_{1}, \ldots, \widehat{m}_{n-1}, \widehat{m}_{n}\right)^{T}$, and $F=\left(f_{0}, f_{1}, \ldots, f_{n-1}, f_{n}\right)^{T}$. After substitution, we get

$$
\begin{aligned}
\hat{M}+ & P \hat{m}+Q \hat{U} \\
= & +\frac{e^{2 \theta}\left(5-7 e^{\theta}\right)}{3\left(-1+e^{\theta}\right)\left(7-18 e^{\theta}+7 e^{2 \theta}\right) \lambda^{2}} A \hat{M}+\frac{e^{2 \theta} \lambda^{2}\left(-80+28 e^{\theta}\right)}{3\left(-1+e^{\theta}\right)\left(7-18 e^{\theta}+7 e^{2 \theta}\right) \lambda^{2}} A \hat{U} \\
& +\frac{\left(-5+7 e^{-\theta}\right)}{3\left(-1+e^{\theta}\right)\left(7-18 e^{\theta}+7 e^{2 \theta}\right) \lambda^{2}} B \hat{M}+\frac{\lambda^{2}\left(80-28 e^{-\theta}\right)}{3\left(-1+e^{\theta}\right)\left(7-18 e^{\theta}+7 e^{2 \theta}\right) \lambda^{2}} B \hat{U} \\
& +\frac{e^{\theta}\left(-8+7 e^{\theta}+7 e^{2 \theta}\right)}{3\left(-1+e^{\theta}\right)\left(7-18 e^{\theta}+7 e^{2 \theta}\right) \lambda^{2}} C \hat{M}+\frac{e^{\theta} \lambda^{2}\left(128-7 e^{\theta}-7 e^{2 \theta}\right)}{3\left(-1+e^{\theta}\right)\left(7-18 e^{\theta}+7 e^{2 \theta}\right) \lambda^{2}} C \hat{U} \\
& +\frac{e^{\theta}\left(-8+7 e^{\theta}+7 e^{2 \theta}\right)}{3\left(-1+e^{\theta}\right)\left(7-18 e^{\theta}+7 e^{2 \theta}\right) \lambda^{2}} D \hat{M}+\frac{e^{\theta} \lambda^{2}\left(128-7 e^{\theta}-7 e^{2 \theta}\right)}{3\left(-1+e^{\theta}\right)\left(7-18 e^{\theta}+7 e^{2 \theta}\right) \lambda^{2}} D \hat{U} \\
& +\frac{8-7 e^{-\theta}-7 e^{-2 \theta}}{3\left(-1+e^{\theta}\right)\left(7-18 e^{\theta}+7 e^{2 \theta}\right) \lambda^{2}} \bar{E} \hat{M}+\frac{\lambda^{2}\left(-128+7 e^{-\theta}+7 e^{-2 \theta}\right)}{3\left(-1+e^{\theta}\right)\left(7-18 e^{\theta}+7 e^{2 \theta}\right) \lambda^{2}} \bar{E} \hat{U} \\
& +\frac{-1-e^{-\theta}+4 e^{-2 \theta}}{\left(-1+e^{\theta}\right)\left(7-18 e^{\theta}+7 e^{2 \theta}\right) \lambda^{2}} R \hat{M}+\frac{\lambda^{2}\left(16+16 e^{-\theta}-4 e^{-2 \theta}\right)}{\left(-1+e^{\theta}\right)\left(7-18 e^{\theta}+7 e^{2 \theta}\right) \lambda^{2}} R \hat{U} \\
& +\frac{-3+5 e^{\theta}}{3\left(-1+e^{\theta}\right)\left(7-18 e^{\theta}+7 e^{2 \theta}\right) \lambda^{2}} G \hat{M}+\frac{\lambda^{2}\left(27-5 e^{\theta}\right)}{3\left(-1+e^{\theta}\right)\left(7-18 e^{\theta}+7 e^{2 \theta}\right) \lambda^{2}} G \hat{U} \\
& +\frac{e^{-\theta}\left(3-5 e^{-\theta}\right)}{3\left(-1+e^{\theta}\right)\left(7-18 e^{\theta}+7 e^{2 \theta}\right) \lambda^{2}} H \hat{M}
\end{aligned}
$$


By solving the above system, an approximation solution of equation (1) will be gotten. Now, the $u_{i}$ function can be approximated by using the exponential spline $\widehat{S}_{i}$, where

$$
\begin{aligned}
\widehat{S}_{i}(x)= & \left(e ^ { \lambda ( x - x _ { i } ) } \left(5 e^{2 \theta} \widehat{M}_{i}-7 e^{3 \theta} \widehat{M}_{i}-5 \widehat{M}_{i+1}+7 e^{-\theta} \widehat{M}_{i+1}-80 e^{2 \theta} \lambda^{2} \widehat{u}_{i}+28 e^{3 \theta} \lambda^{2} \widehat{u}_{i}\right.\right. \\
& \left.\left.+80 \lambda^{2} \widehat{u}_{i+1}-28 e^{-\theta} \lambda^{2} \widehat{u}_{i+1}\right)\right) \\
& /\left(3\left(-1+e^{\theta}\right)\left(7-18 e^{\theta}+7 e^{2 \theta}\right) \lambda^{2}\right) \\
& +\left(e ^ { 4 \lambda ( x - x _ { i } ) } \left(-3 \widehat{M}_{i}+5 e^{\theta} \widehat{M}_{i}-5 e^{-2 \theta} \widehat{M}_{i+1}+3 e^{-\theta} \widehat{M}_{i+1}+27 \lambda^{2} \widehat{u}_{i}\right.\right. \\
& \left.\left.-5 e^{\theta} \lambda^{2} \widehat{u}_{i}+5 e^{-2 \theta} \lambda^{2} \widehat{u}_{i+1}-27 e^{-\theta} \lambda^{2} \widehat{u}_{i+1}\right)\right) \\
& /\left(3\left(-1+e^{\theta}\right)\left(7-18 e^{\theta}+7 e^{2 \theta}\right) \lambda^{2}\right) \\
& +\left(e ^ { 2 \lambda ( x - x _ { i } ) } \left(-8 e^{\theta} \widehat{M}_{i}+7 e^{2 \theta} \widehat{M}_{i}+7 e^{3 \theta} \widehat{M}_{i}+8 \widehat{M}_{i+1}-7 e^{-2 \theta} \widehat{M}_{i+1}-7 e^{-\theta} \widehat{M}_{i+1}\right.\right. \\
& \left.\left.+128 \lambda^{2} e^{\theta} \widehat{u}_{i}-7 e^{2 \theta} \lambda^{2} \widehat{u}_{i}\right)\right) \\
& /\left(3\left(-1+e^{\theta}\right)\left(7-18 e^{\theta}+7 e^{2 \theta}\right) \lambda^{2}\right) \\
& +\frac{e^{2 \lambda\left(x-x_{i}\right)}\left(-7 \lambda^{2} e^{3 \theta} \widehat{u}_{i}-128 \lambda^{2} \widehat{u}_{i+1}+7 e^{-2 \theta} \lambda^{2} \widehat{u}_{i+1}+7 e^{-\theta} \lambda^{2} \widehat{u}_{i+1}\right)}{3\left(-1+e^{\theta}\right)\left(7-18 e^{\theta}+7 e^{2 \theta}\right) \lambda^{2}} \\
& +\left(e ^ { 3 \lambda ( x - x _ { i } ) } \left(\widehat{M}_{i}+e^{\theta} \widehat{M}_{i}-4 e^{2 \theta} \widehat{M}_{i}-\widehat{M}_{i+1}+4 e^{-2 \theta} \widehat{M}_{i+1}-e^{-\theta} \widehat{M}_{i+1}\right.\right. \\
& \left.\left.-16 \lambda^{2} \widehat{u}_{i}-16 e^{\theta} \lambda^{2} \widehat{u}_{i}\right)\right) \\
& /\left(\left(-1+e^{\theta}\right)\left(7-18 e^{\theta}+7 e^{2 \theta}\right) \lambda^{2}\right) \\
& +\frac{e^{3 \lambda\left(x-x_{i}\right)}\left(+4 \lambda^{2} e^{2 \theta} \widehat{u}_{i}+16 \lambda^{2} \widehat{u}_{i+1}+4 e^{-2 \theta} \lambda^{2} \widehat{u}_{i+1}+16 e^{-\theta} \lambda^{2} \widehat{u}_{i+1}\right)}{\left(-18 e^{\theta}+7 e^{2 \theta}\right) \lambda^{2}}+O\left(h^{4}\right) \\
& =(3-1) \\
&
\end{aligned}
$$

In consequence, for all $i=0(1) n-1$ and $x \in\left(x_{i}, x_{i+1}\right)$, we get

$$
\left|S_{i}(x)-\widehat{S_{i}}(x)\right| \equiv \kappa_{0} h^{4}
$$

and similarly we get

$$
\left|S_{i}^{\prime \prime}(x)-\widehat{S_{i}^{\prime \prime}}(x)\right| \equiv \kappa_{1} h^{2}
$$

See [38].

\section{Convergence of the method}

In this section the convergence of the method is proved. To do this, we consider equation (10) in a matrix form as follows:

$$
\begin{aligned}
\hat{M}+ & P \hat{m}+Q \hat{U} \\
= & F+\eta e^{2 \theta}\left(5-7 e^{\theta}\right) A \hat{M}+\eta e^{2 \theta} \lambda^{2}\left(-80+28 e^{\theta}\right) A \hat{U} \\
& +\eta\left(-5+7 e^{-\theta}\right) B \hat{M}+\eta \lambda^{2}\left(80-28 e^{-\theta}\right) B \hat{U} \\
& +\eta e^{\theta}\left(-8+7 e^{\theta}+7 e^{2 \theta}\right) C \hat{M}+\eta e^{\theta} \lambda^{2}\left(128-7 e^{\theta}-7 e^{2 \theta}\right) C \hat{U} \\
& +\eta e^{\theta}\left(-8+7 e^{\theta}+7 e^{2 \theta}\right) D \hat{M}+\eta e^{\theta} \lambda^{2}\left(128-7 e^{\theta}-7 e^{2 \theta}\right) D \hat{U}
\end{aligned}
$$




$$
\begin{aligned}
& +\eta\left(8-7 e^{-\theta}-7 e^{-2 \theta}\right) \bar{E} \hat{M}+\eta \lambda^{2}\left(-128+7 e^{-\theta}+7 e^{-2 \theta}\right) \bar{E} \hat{U} \\
& +\eta\left(-1-e^{-\theta}+4 e^{-2 \theta}\right) R \hat{M}+\eta \lambda^{2}\left(16+16 e^{-\theta}-4 e^{-2 \theta}\right) R \hat{U} \\
& +\eta\left(-3+5 e^{\theta}\right) G \hat{M}+\eta \lambda^{2}\left(27-5 e^{\theta}\right) G \hat{U} \\
& +\eta e^{-\theta}\left(3-5 e^{-\theta}\right) H \hat{M}+\eta e^{-\theta} \lambda^{2}\left(-27+5 e^{-\theta}\right) H \hat{U},
\end{aligned}
$$

where

$$
\eta=\frac{1}{3\left(-1+e^{\theta}\right)\left(7-18 e^{\theta}+7 e^{2 \theta}\right) \lambda^{2}} .
$$

Using (14), we get the following expression:

$$
\begin{aligned}
\widehat{M}+ & P \widehat{m}+Q \widehat{U} \\
= & +\eta\left(e^{2 \theta}\left(5-7 e^{\theta}\right) A+\left(-5+7 e^{-\theta}\right) B+e^{\theta}\left(-8+7 e^{\theta}+7 e^{2 \theta}\right) C\right. \\
& \left.+\eta e^{\theta}\left(-8+7 e^{\theta}+7 e^{2 \theta}\right) D\right) \widehat{M} \\
& +\eta\left(\left(8-7 e^{-\theta}-7 e^{-2 \theta}\right) \bar{E}+\left(-1-e^{-\theta}+4 e^{-2 \theta}\right) R+\left(-3+5 e^{\theta}\right) G\right. \\
& \left.+e^{-\theta}\left(3-5 e^{-\theta}\right) H\right) \widehat{M} \\
& +\eta \lambda^{2}\left(e^{2 \theta}\left(-80+28 e^{\theta}\right) A+\left(80-28 e^{-\theta}\right) B+e^{\theta}\left(128-7 e^{\theta}-7 e^{2 \theta}\right) C\right. \\
& \left.+e^{\theta}\left(128-7 e^{\theta}-7 e^{2 \theta}\right) D\right) \widehat{U} \\
& +\eta \lambda^{2}\left(\left(-128+7 e^{-\theta}+7 e^{-2 \theta}\right) \bar{E}+\left(16+16 e^{-\theta}-4 e^{-2 \theta}\right) R+\left(27-5 e^{\theta}\right) G\right. \\
& \left.+e^{-\theta}\left(-27+5 e^{-\theta}\right) H\right) \widehat{U} \\
\Rightarrow & W^{-1} \bar{R} \widehat{U}+P Z^{-1} \widehat{U}+Q \widehat{U}=F+H_{1} W^{-1} \widehat{R} \widehat{U}+H_{2} \widehat{U},
\end{aligned}
$$

where

$$
\begin{aligned}
H_{1}= & \eta e^{2 \theta}\left(5-7 e^{\theta}\right) A+\eta\left(-5+7 e^{-\theta}\right) B+\eta e^{\theta}\left(-8+7 e^{\theta}+7 e^{2 \theta}\right) C \\
& +\eta e^{\theta}\left(-8+7 e^{\theta}+7 e^{2 \theta}\right) D+\eta\left(8-7 e^{-\theta}-7 e^{-2 \theta}\right) \bar{E} \\
& +\eta\left(-1-e^{-\theta}+\eta 4 e^{-2 \theta}\right) R+\eta\left(-3+5 e^{\theta}\right) G+\eta e^{-\theta}\left(3-5 e^{-\theta}\right) H, \\
H_{2}= & \eta \lambda^{2}\left(e^{2 \theta}\left(-80+28 e^{\theta}\right) A+\eta\left(80-28 e^{-\theta}\right) B\right. \\
& \left.+\eta e^{\theta}\left(128-7 e^{\theta}-7 e^{2 \theta}\right) C+\eta e^{\theta}\left(128-7 e^{\theta}-7 e^{2 \theta}\right) D\right) \\
& +\eta \lambda^{2}\left(\left(-128+7 e^{-\theta}+7 e^{-2 \theta}\right) \bar{E}+\eta\left(16+16 e^{-\theta}-4 e^{-2 \theta}\right) R\right. \\
& \left.+\eta\left(27-5 e^{\theta}\right) G+\eta e^{-\theta}\left(-27+5 e^{-\theta}\right) H\right) .
\end{aligned}
$$

So the exact solution can be written as follows:

$$
Q\left[I-\left(-Q^{-1} W^{-1} \bar{R}-Q^{-1} P Z^{-1} S+Q^{-1} H_{1} W^{-1} \bar{R}+Q^{-1} H_{2}\right)\right] \bar{U}=F+T,
$$

where $\bar{U}=\left[u\left(x_{0}\right), u\left(x_{1}\right), \ldots, u\left(x_{n}\right)\right]^{T}$ is the $(\mathrm{n}+1)$-dimensional column vector of the exact solution, the vector of local truncation error is displayed as $T=\left[t_{0}, t_{2}, \ldots, t_{n}\right]^{T}$. According 
to (15) and (16), we have

$$
Q\left[I-\left(-Q^{-1} W^{-1} \bar{R}-Q^{-1} P Z^{-1} S+Q^{-1} H_{1} W^{-1} \bar{R}+Q^{-1} H_{2}\right)\right] E=T,
$$

where $E=\left(e_{j}\right)$ indicates the column vector of $e_{i}, i=0,1,2, \ldots, n$, which is $(n+1)$ dimensional. Since $A_{n \times n}$ is a diagonally-dominant matrix, then $\left|A_{n \times n}\right| \neq 0$. We need the following lemma for analysis of convergence.

Lemma 1 Let $N$ be an $n \times n$ matrix with $\|N\|_{\infty}<1$. So, the matrix $(I-N)$ is invertible. Moreover, $\left\|(I-N)^{-1}\right\|_{\infty} \leq \frac{1}{1-\|N\|_{\infty}}$.

Lemma 2 The matrices $W$ and $Z$ are invertible.

Proof For $\alpha=\frac{1}{6}, \beta=\frac{1}{3}$ and $\alpha=\frac{1}{12}, \beta=\frac{5}{12}$, the matrices $W$ and $Z$ are diagonally-dominant matrices, then are invertible. By using the inversion of general tridiagonal matrices [16] and [8], it is easy to prove that $\left\|W^{-1}\right\|_{\infty} \leq 1$ for $\alpha=\frac{1}{12}, \beta=\frac{5}{12}$ and $\left\|Z^{-1}\right\|_{\infty} \leq 1$ for $\alpha=\frac{1}{6}$, $\beta=\frac{1}{3}$. We need to show that the inverse of $Q\left[I-\left(-Q^{-1} W^{-1} \bar{R}-Q^{-1} P Z^{-1} S+Q^{-1} H_{1} W^{-1} \bar{R}+\right.\right.$ $\left.Q^{-1} H_{2}\right)$ ] exists. Now, if $Q$ is a diagonal matrix with the inverse $Q^{-1}$, we can derive the following lemma. We obtain $\left\|Q^{-1}\right\|_{\infty} \leq \frac{1}{\max \left|q_{i i}\right|}=\xi$.

Lemma 3 The matrix $\left[I-\left(-Q^{-1} W^{-1} \bar{R}-Q^{-1} P Z^{-1} S+Q^{-1} H_{1} W^{-1} \bar{R}+Q^{-1} H_{2}\right)\right]$ is nonsingular, provided

$$
\xi\left(\eta_{2} h^{2}+\eta_{1} \eta_{3} h^{2}+\eta_{2} \eta_{5}\|k\|_{\infty}(b-a) h^{4}+\|k\|_{\infty}(b-a) \eta_{6}\right)<1 .
$$

Proof Obviously, for $i=0,1, \ldots, n$, it can be verified as follows:

$$
\left\{\begin{array}{l}
\|A\|_{\infty}=\|B\|_{\infty} \leq\|k\|_{\infty}(b-a)\left(\frac{e^{\theta}-1}{\theta}\right) \\
\|C\|_{\infty}=\|D\|_{\infty} \leq\|k\|_{\infty}(b-a)\left(\frac{e^{\theta \theta}-1}{2 \theta}\right) \\
\|E\|_{\infty}=\|R\|_{\infty} \leq\|k\|_{\infty}(b-a)\left(\frac{e^{3 \theta}-1}{3 \theta}\right) \\
\|G\|_{\infty}=\|H\|_{\infty} \leq\|k\|_{\infty}(b-a)\left(\frac{e^{\theta \theta}-1}{4 \theta}\right) \\
\|P\|_{\infty}=\operatorname{Max}\left|p\left(x_{i}\right)\right| \leq \eta_{3} \\
\|Q\|_{\infty}=\operatorname{Max}\left|q\left(x_{i}\right)\right| \leq \eta_{4} \\
\|S\|_{\infty} \leq \eta_{1} h^{2},\|\bar{R}\|_{\infty} \leq \eta_{2} h^{2} \\
\left\|H_{1}\right\|_{\infty} \leq\|k\|_{\infty}(b-a) h^{2} \eta_{5} \\
\left\|H_{2}\right\|_{\infty} \leq\|k\|_{\infty}(b-a) \eta_{6}
\end{array}\right.
$$

where

$$
\begin{aligned}
\eta_{5}= & \left|\frac{1}{3\left(e^{\theta}-1\right)\left(-18 e^{\theta}+7 e^{2 \theta}+7\right) \theta^{2}}\right|\left(\left|\frac{e^{2 \theta}\left(5-7 e^{\theta}\right)\left(e^{\theta}-1\right)}{\theta}\right|+\left|\frac{\left(7 e^{-\theta}-5\right)\left(e^{\theta}-1\right)}{\theta}\right|\right. \\
& +\left|\frac{e^{\theta}\left(7 e^{\theta}+7 e^{2 \theta}-8\right)\left(e^{2 \theta}-1\right)}{2 \theta}\right|+\left|\frac{e^{\theta}\left(7 e^{\theta}+7 e^{2 \theta}-8\right)\left(e^{2 \theta}-1\right)}{2 \theta}\right| \\
& +\left|\frac{\left(-7 e^{-\theta}-7 e^{-2 \theta}+8\right)\left(e^{3 \theta}-1\right)}{3 \theta}\right|+\left|\frac{\left(-e^{-\theta}+4 e^{-2 \theta}-1\right)\left(e^{3 \theta}-1\right)}{3 \theta}\right| \\
& \left.+\left|\frac{\left(5 e^{\theta}-3\right)\left(e^{4 \theta}-1\right)}{4 \theta}\right|+\left|\frac{e^{-\theta}\left(3-5 e^{-\theta}\right)\left(e^{4 \theta}-1\right)}{4 \theta}\right|\right),
\end{aligned}
$$




$$
\begin{aligned}
\eta_{6}= & \left|\frac{1}{3\left(e^{\theta}-1\right)\left(-18 e^{\theta}+7 e^{2 \theta}+7\right)}\right|\left(\left|\frac{e^{2 \theta}\left(28 e^{\theta}-80\right)\left(e^{\theta}-1\right)}{\theta}\right|+\left|\frac{\left(80-28 e^{-\theta}\right)\left(e^{\theta}-1\right)}{\theta}\right|\right. \\
& +\left|\frac{e^{\theta}\left(-7 e^{\theta}-7 e^{2 \theta}+128\right)\left(e^{2 \theta}-1\right)}{2 \theta}\right|+\left|\frac{e^{\theta}\left(-7 e^{\theta}-7 e^{2 \theta}+128\right)\left(e^{2 \theta}-1\right)}{2 \theta}\right| \\
& +\left|\frac{\left(7 e^{-\theta}+7 e^{-2 \theta}-128\right)\left(e^{3 \theta}-1\right)}{3 \theta}\right|+\left|\frac{\left(16 e^{-\theta}-4 e^{-2 \theta}+16\right)\left(e^{3 \theta}-1\right)}{3 \theta}\right| \\
& \left.+\left|\frac{\left(27-5 e^{\theta}\right)\left(e^{4 \theta}-1\right)}{4 \theta}\right|+\left|\frac{e^{-\theta}\left(5 e^{-\theta}-27\right)\left(e^{4 \theta}-1\right)}{4 \theta}\right|\right) .
\end{aligned}
$$

By using Lemma 1, we get

$$
\begin{aligned}
& \left\|Q^{-1}\right\|\left(\left\|W^{-1}\right\|\|\bar{R}\|+\|P\|\left\|Z^{-1}\right\|\|S\|+\left\|H_{1}\right\|\left\|W^{-1}\right\|\|\bar{R}\|+\left\|H_{2}\right\|\right)<1, \\
& \xi\left(\eta_{2} h^{2}+\eta_{1} \eta_{3} h^{2}+\eta_{2} \eta_{5}\|k\|_{\infty}(b-a) h^{4}+\|k\|_{\infty}(b-a) \eta_{6}\right)<1 .
\end{aligned}
$$

Theorem 1 Assume $f(x) \in C^{4}(I), k(t, x) \in C^{4}(I \times I)$ in a way that

$$
\xi\left(\eta_{2} h^{2}+\eta_{1} \eta_{3} h^{2}+\eta_{2} \eta_{5}\|k\|_{\infty}(b-a) h^{4}+\|k\|_{\infty}(b-a) \eta_{6}\right)<1
$$

Therefore consider a unique approximating solution and the obtained error $E:=U-\hat{S}$ satisfies

$$
\|E\| \equiv O\left(h^{2}\right)
$$

where $\Omega:=[a, b]$; moreover, $\alpha, \eta_{l}$ for $l=1,2,3,4,5,6$ are constants.

Proof By using equation (17) and Lemma 1, we get

$$
\|E\| \leq \frac{\left\|Q^{-1}\right\|\|T\|}{1-\left\|Q^{-1}\right\|\left(\left\|W^{-1}\right\|\|\bar{R}\|+\|P\|\left\|Z^{-1}\right\|\|S\|+\left\|H_{1}\right\|\left\|W^{-1}\right\|\|\bar{R}\|+\left\|H_{2}\right\|\right)} .
$$

By substituting $\|T\| \leq \frac{h^{6}}{240} \phi_{4}$ and (18) in (19), we get

$$
\|E\| \equiv O\left(h^{2}\right)
$$

Therefore, we have

$$
\|U-\hat{S}\|_{\infty} \leq \zeta_{2} h^{2}
$$

Therefore, applying (12) and (20) leads to

$$
\|U-\hat{S}\|_{\infty} \leq\|U-S\|_{\infty}+\|S-\hat{S}\|_{\infty} \leq \zeta_{2} h^{2}+\zeta_{0} h^{4} \equiv O\left(h^{2}\right)
$$

Then it may follow $\|E\| \rightarrow 0$ if $h \rightarrow 0$. So, for $\left(\alpha=\frac{1}{12}, \beta=\frac{5}{12}\right)$ and $\left(\alpha=\frac{1}{6}, \beta=\frac{1}{3}\right)$, we established the convergence of second-order method because we approximated $m_{0}, m_{n}, M_{0}$, and $M_{n}$ by second-order methods. Therefore, $\alpha$ and $\beta$ do not affect the second order of convergence. 


\section{Numerical results}

Here, we apply our method for $\alpha=\frac{1}{12}, \beta=\frac{5}{12}$ on some examples of the second-order boundary value problems of Fredholm integro-differential equation. First, the absolute error is calculated and then compared with the well-known methods in [11]. Note that numerical results are derived by MAPLE 14 .

Example 1 As the first example, consider the following boundary value problem:

$$
u^{\prime \prime}(x)+x u^{\prime}(x)+\pi^{2} u(x)=f(x)+\int_{0}^{1} k(t, x) u(t) d x, \quad x \in[0,1],
$$

subject to boundary conditions

$$
u(0)=u(1)=0,
$$

where $k(t, x)=x+t, f(x)=\pi x \cos (\pi x)-\frac{2 x+1}{\pi}$, and $u(x)=\sin (\pi x)$ is exact solution. The absolute errors are presented in Tables 1 and 2. The convergence ratio (C.R.) is gotten as follows:

$$
\text { C.R. }=\log _{2} \frac{E(h)}{E\left(\frac{h}{2}\right)},
$$

where the maximum absolute error is shown by $E(h)$.

Example 2 Consider the following boundary value problem [11]:

$$
u^{\prime \prime}(x)=f(x)+\int_{0}^{1} k(t, x) u(t) d x, \quad x \in[0,1]
$$

with boundary conditions

$$
u(0)=u(1)=0,
$$

Table $1 E(h)$ of Example 1

\begin{tabular}{rllll}
\hline $\mathrm{n}$ & Our method & C.R. & Method in [11] & C.R \\
\hline 16 & $1.2194 \mathrm{e}-3$ & & $1.6002 \mathrm{e}-2$ & \\
32 & $3.7902 \mathrm{e}-4$ & 1.6858 & $4.0613 \mathrm{e}-3$ & 1.98 \\
64 & $1.0683 \mathrm{e}-4$ & 1.8271 & $1.0192 \mathrm{e}-3$ & 1.99 \\
128 & $2.8367 \mathrm{e}-5$ & 1.9131 & $2.5504 \mathrm{e}-4$ & 1.99 \\
\hline
\end{tabular}

Table 2 Comparison of solutions obtained by the presented method with the exact solution of Example 1 while $n=128$

\begin{tabular}{llll}
\hline$x$ & Exact solution & Approximating solution & Obtained errors \\
\hline 0.125 & 0.382683 & 0.382694 & $1.11 \mathrm{e}-5$ \\
0.250 & 0.707107 & 0.707027 & $2.07 \mathrm{e}-5$ \\
0.375 & 0.923879 & 0.923906 & $2.67 \mathrm{e}-5$ \\
0.500 & 1.000000 & 1.000028 & $2.83 \mathrm{e}-5$ \\
0.625 & 0.923880 & 0.923904 & $2.54 \mathrm{e}-5$ \\
0.750 & 0.707107 & 0.707125 & $1.88 \mathrm{e}-5$ \\
0.875 & 0.382683 & 0.382693 & $0.98 \mathrm{e}-5$ \\
\hline
\end{tabular}


where $k(t, x)=e^{x t}, f(x)=2+\frac{x+2+e^{x}(x-2)}{x^{3}}$, and $u(x)=x(x-1)$ is the exact solution. The absolute errors are presented in Tables 3 and 4 .

Example 3 Consider the following boundary value problem:

$$
u^{\prime \prime}(x)-u^{\prime}(x)=f(x)+\int_{0}^{1} k(t, x) u(t) d x, \quad x \in[0,1]
$$

subject to boundary conditions

$$
u(0)=u(1)=0,
$$

where $k(t, x)=x t, f(x)=\frac{1+41 x^{3}+31 x^{5}-46 x^{4}+8 x-25 x^{2}-13 x^{6}+2 x^{7}}{\left(x^{2}-x+1\right)^{2}}+\frac{121}{120} x-\frac{1}{6} \sqrt{3} \pi x$, and $u(x)=$ $\ln \left(x^{2}-x+1\right)-\frac{\left(x^{2}-x\right)^{2}}{2}$ is the exact solution. The absolute errors are presented in Tables 5 and 6 .

Table $3 E(h)$ of Example 2

\begin{tabular}{rllll}
\hline$n$ & Our method & C.R. & Best in [11] & C.R \\
\hline 16 & $6.1104 \mathrm{e}-4$ & & $6.5606 \mathrm{e}-4$ & \\
32 & $1.5751 \mathrm{e}-4$ & 1.9558 & $1.6398 \mathrm{e}-4$ & 2.0003 \\
64 & $4.0018 \mathrm{e}-5$ & 1.9767 & $4.0991 \mathrm{e}-5$ & 2.0001 \\
128 & $1.0087 \mathrm{e}-5$ & 1.9882 & $1.0248 \mathrm{e}-5$ & 2.0000 \\
\hline
\end{tabular}

Table 4 Comparison of solutions obtained by the presented method with the exact solution of Example 2 while $n=128$

\begin{tabular}{llll}
\hline$x$ & Exact solution & Approximating solution & Obtained errors \\
\hline 0.125 & $-1.09367 \mathrm{e}-1$ & $-1.09375 \mathrm{e}-1$ & $8.00000 \mathrm{e}-6$ \\
0.250 & $-1.87492 \mathrm{e}-1$ & $-1.87500 \mathrm{e}-1$ & $8.00000 \mathrm{e}-6$ \\
0.375 & $-2.34367 \mathrm{e}-1$ & $-2.34375 \mathrm{e}-1$ & $8.00000 \mathrm{e}-6$ \\
0.500 & $-2.49993 \mathrm{e}-1$ & $-2.50000 \mathrm{e}-1$ & $7.00000 \mathrm{e}-6$ \\
0.625 & $-2.34368 \mathrm{e}-1$ & $-2.34375 \mathrm{e}-1$ & $7.00000 \mathrm{e}-6$ \\
0.750 & $-1.87492 \mathrm{e}-1$ & $-1.87500 \mathrm{e}-1$ & $8.00000 \mathrm{e}-6$ \\
0.875 & $-1.09366 \mathrm{e}-1$ & $-1.09375 \mathrm{e}-1$ & $9.00000 \mathrm{e}-6$ \\
\hline
\end{tabular}

Table $5 E(h)$ of Example 3

\begin{tabular}{rll}
\hline$n$ & Our method & C.R. \\
\hline 16 & $2.0224 \mathrm{e}-3$ & \\
32 & $5.3502 \mathrm{e}-4$ & 1.9184 \\
64 & $1.3644 \mathrm{e}-4$ & 1.9713 \\
128 & $3.4381 \mathrm{e}-5$ & 1.9886 \\
\hline
\end{tabular}

Table 6 Comparison of solutions obtained by the presented method with the exact solution of Example 3 while $n=128$

\begin{tabular}{llll}
\hline$x$ & Exact solution & Approximating solution & Obtained errors \\
\hline 0.125 & $-1.2181 \mathrm{e}-1$ & $-1.2184 \mathrm{e}-1$ & $3.000 \mathrm{e}-5$ \\
0.250 & $-2.2522 \mathrm{e}-1$ & $-2.2524 \mathrm{e}-1$ & $2.000 \mathrm{e}-5$ \\
0.375 & $-2.9453 \mathrm{e}-1$ & $-2.9456 \mathrm{e}-1$ & $3.000 \mathrm{e}-5$ \\
0.500 & $-3.1893 \mathrm{e}-1$ & $-3.1895 \mathrm{e}-1$ & $2.000 \mathrm{e}-5$ \\
0.625 & $-2.9454 \mathrm{e}-1$ & $-2.9455 \mathrm{e}-1$ & $1.000 \mathrm{e}-5$ \\
0.750 & $-2.2522 \mathrm{e}-1$ & $-2.2523 \mathrm{e}-1$ & $1.000 \mathrm{e}-5$ \\
0.875 & $-1.2181 \mathrm{e}-1$ & $-1.2182 \mathrm{e}-1$ & $1.000 \mathrm{e}-5$ \\
\hline
\end{tabular}




\section{Conclusion}

In our knowledge, so far the exponential spline functions have not been yet applied for approximating the second-order integro-differential equations. In this study, according to the exponential method in [31], a suitable method is presented to approximate secondorder integro-differential equations. The proposed algorithm is novel for second-order integro-differential equations. The second-order convergence of the proposed method has been derived and the computational outcomes have been found to be conformable with theoretical expectations. Our method shows better accuracy compared to the existing method in [11].

\section{Acknowledgements}

The authors are grateful to the reviewers for their helpful, valuable comments and suggestions in the improvement of this manuscript.

\section{Funding}

Not applicable.

\section{Abbreviations}

Not applicable.

Availability of data and materials

Not applicable.

\section{Competing interests}

The authors declare that they have no competing interests.

Authors' contributions

All authors contributed equally to this article. All authors read and approved the final manuscript.

\section{Publisher's Note}

Springer Nature remains neutral with regard to jurisdictional claims in published maps and institutional affiliations.

Received: 18 August 2019 Accepted: 17 March 2020 Published online: 31 March 2020

\section{References}

1. Amirfakhrian, M., Shakibi, K.: Solving integro-differential equation by using b-spline interpolation. Int. J. Math. Model. Comput. 3, 237-244 (2013)

2. Balci, M.A., Sezer, M.: A numerical approach based on exponential polynomials for solving of Fredholm integro-differential-difference equations. New Trends Math. Sci. 3(2), 44-54 (2015)

3. Bashan, A:: An effective application of differential quadrature method based on modified cubic B-splines to numerical solutions of the KdV equation. Turk. J. Math. 42, 373-394 (2018)

4. Basirat, B., Shahdadi, M.A.: Numerical solution of nonlinear integro-differential equations with initial conditions by Bernstein operational matrix of derivative. Int. J. Mod. Nonlinear Theory Appl. 2, 141-149 (2013)

5. Behiry, S.H.: Solution of nonlinear Fredholm integro-differential equations using a hybrid of block pulse functions and normalized Bernstein polynomials. J. Comput. Appl. Math. 260, 258-265 (2014)

6. Behiry, S.H., Hashish, H.: Wavelet methods for the numerical solution of Fredholm integro-differential equations. Int. J. Appl. Math. 11, 27-35 (2002)

7. Behiry, S.H., Mohamed, S.I.: Solving high-order nonlinear Volterra-Fredholm integro-differential equations by differential trasform method. Nat. Sci. 4(8), 581-587 (2012)

8. Belloura, A., Sbibih, D., Zidna, A.: Two cubic spline methods for solving Fredholm integral equations. Appl. Math. Comput. 276, 1-11 (2016)

9. Bhrawy, A.H., Tohidi, E., Soleymani, F.: A new Bernoulli matrix method for solving high-order linear and nonlinear Fredholm integro-differential equations with piecewise intervals. Appl. Math. Comput. 219, 482-497 (2012)

10. Brunner, H.: On the numerical solution of Volterra-Fredholm integral equation by collocation methods. SIAM J. Numer. Anal. 27, 87-96 (1990)

11. Chen, J., Huang, Y., Rong, H., Wu, T., Zeng, T.: A multiscale Galerkin method for second-order boundary value problems of Fredholm integro-differential equation. J. Comput. Appl. Math. 290, 633-640 (2015)

12. Cravero, L., Pittaluga, G., Sacripante, L.: An algorithm for solving linear Volterra integro-differential equations. Numer. Algorithms 60, 101-114 (2012)

13. Dehghan, M., Saadatmandi, A.: Chebyshev finite difference method for Fredholm integro-differential equation. Int. J. Comput. Math. 85, 123-130 (2008)

14. Ding, Q., Wong, P.J.Y.: Mid-knot cubic non-polynomial spline for a system of second-order boundary value problems. Bound. Value Probl. 2018, Article ID 156 (2018)

15. Dursun, I., Keskin Yildiz, P., Zorşahin Görgülü, M., Quartic trigonometric B-spline algorithm for numerical solution of the regularized long wave equation. Turk. J. Math. 43, 112-125 (2019) 
16. El-Mikkawy, M., Karawia, A.: Inversion of general tridiagonal matrices. Appl. Math. Lett. 19, 715-720 (2006)

17. Ersoy, O., Dag, l.: The extended B-spline collocation method for numerical solutions of Fisher equation. AIP Conf. Proc 1648, Article ID 370011 (2015)

18. Ersoy, O., Dag, l.: The exponential cubic b-spline collocation method for the Kuramoto-Sivashinsky equation. Filomat 30(3), 853-861 (2016)

19. Feng, M., Pang, H.: A class of three point boundary value problems for second-order impulsive integro-differential equations in Banach space. Nonlinear Anal. 70, 64-82 (2009)

20. Firouzdor, R., Heidarnejad, K.A., Mollarason, M.Z.: Numerical solution of functional integral equations by using B-splines. J. Linear Topol. Algebra 1, 45-53 (2012)

21. Ghoreishi, F., Hadizadeh, M.: Numerical computation of the tau approximation for the Volterra-Hammerstein integral equations. Numer. Algorithms 52, 541-559 (2009)

22. Han, D.F., Shang, X.F.: Numerical solution of integro-differential equations by using CAS wavelet operational matrix of integration. Appl. Math. Comput. 156, 460-466 (2007)

23. Hepson, O.E.: Generation of the trigonometric cubic b-spline collocation solutions fort he Kuramoto-Sivashinsky (KS) equation. AIP Conf. Proc. 1978, Article ID 470099 (2018)

24. Hepson, O.E., Korkmaz, A., Dag, I.: Numerical solutions of the Gardner equation by extended form of the cubic b-splines. Pramana J. Phys. 91, Article ID 59 (2018)

25. Ishtiaq, A.: Jacobi-spectral method for integro-delay differential equations with weakly singular kernels. Turk. J. Math. 39, 810-819 (2015)

26. Li, X., Wong, P.J.Y.: A higher order non-polynomial spline method for fractional sub-diffusion problems. J. Comput. Phys. 328, 46-65 (2017)

27. Li, X., Wong, P.J.Y.: An efficient non-polynomial spline method for distributed order fractional sub-diffusion equations Math. Methods Appl. Sci. 41(13), 4906-4922 (2018)

28. Li, X., Wong, P.J.Y.: Non-polynomial spline approach in two-dimensional fractional sub-diffusion problems. Appl. Math. Comput. 357, 222-242 (2019)

29. Li, X., Wong, P.J.Y.: Numerical solutions of fourth-order fractional sub-diffusion problems via parametric quintic spline. Z. Angew. Math. Mech. 99(5), Article ID e201800094 (2019)

30. Mahmoodi, Z., Rashidinia, J., Babolian, E.: B-spline collocation method for linear and nonlinear Fredholm and Volterra integro-differential equations. Appl. Anal. 93, 1787-1802 (2013)

31. Maleknejad, K., Rashidinia, J., Jalilian, H.: Non-polynomial spline basis and quasilinearization method to solve the nonlinear Volterra integral equation. Filomat 32(11), 3947-3956 (2018)

32. Mirzaee, F.: Numerical solution of nonlinear Fredholm-Volterra integral equations via Bell polynomials. Comput. Methods Differ. Equ. 5, 88-102 (2017)

33. Netravali, A.N.: Spline approximation to the solution of the Volterra integral equation of the second kind. Math. Comput. 121, 99-106 (1973)

34. Ogunlaran, O.M., Oke, M.O.: A numerical approach for solving first order integro-differential equations. Am. J. Comput. Appl. Math. 3, 214-219 (2013)

35. Ordokhani, Y.: An application of Walsh functions for Fredholm-Hammerstein integro-differential equations. Int. J. Contemp. Math. Sci. 22, 1055-1063 (2010)

36. Pittaluga, G., Sacripante, L.: A numerical algorithm for cubature by bivariate splines on nonuniform partitions. Numer. Algorithms 28, 273-284 (2001)

37. Pittaluga, G., Sacripante, L.: An algorithm for solving Fredholm integro-differential equations. Numer. Algorithms 50 , 114-126 (2009)

38. Rashidinia, J., Maleknejad, Kh., Jalilian, H.: Convergence analysis of non-polynomial spline functions for the Fredholm integral equation. Int. J. Comput. Math. (2019). https://doi.org/10.1080/00207160.2019.1609669

39. Saadatmandi, A., Dehghan, M.: Numerical solution of high-order linear Fredholm integro-differential-difference equation with variable coefficients. Comput. Math. Appl. 59, 2996-3004 (2010)

40. Sadri, K., Amini, A., Cheng, C.: A new numerical method for delay and advanced integro-differential equations. Numer Algorithms 77, 381-412 (2018)

41. Singh, P., Joshi, S.D., Patney, R.K., Saha, K.: Some studies on nonpolynomial interpolation and error analysis. Appl. Math Comput. 244, 809-821 (2014)

42. Sloan, I.H.: Non-polynomial interpolation. J. Approx. Theory 39, 97-117 (1983)

43. Stoer, J., Bulirsch, R.: Introduction to Numerical Analysis, 2nd edn. Springer, Berlin (1992)

44. Thiem, H.R.: A model for spatio spread of an epidemic. J. Math. Biol. 4, 337-351 (1977)

45. Wang, Z., Liu, L., Wu, Y.: The unique solution of boundary value problems for nonlinear second-order integro-differential equations of mixed type in Banach space. Comput. Math. Appl. 54, 1293-1301 (2007)

46. Yalcinbas, S.: Taylor polynomial solution of nonlinear Volterra-Fredholm integral equations. Appl. Math. Comput. 127 195-206 (2002)

47. Yang, X., Shen, J.: Periodic boundary value problems for second-order impulsive integro-differential equations. J. Comput. Appl. Math. 209, 176-186 (2007)

48. Yeganeh, S., Ordokhani, Y., Saadatmandi, A.: A sinc-collocation method for second-order boundary value problems of nonlinear integro-differential equation. J. Inf. Comput. Sci. 7, 151-160 (2012)

49. Yulan, W., Chaolu, T., Jing, P.: New algorithm for second-order boundary value problems of integro-differential equation. J. Comput. Appl. Math. 229, 1-6 (2009)

50. Yüzbaşi, Ş.: An exponential collocation method for the solutions of the HIV infection model of CD4+ T cells. Int. J. Biomath. 9(3), Article ID 1650036 (2016)

51. Yüzbaşi, Ş.: An exponential method to solve linear Fredholm-Volterra integro-differential equations and residual improvement. Turk. J. Math. 42, 2546-2562 (2018)

52. Yüzbași, S., Karaçayir, M.: An exponential Galerkin method for solutions of HIV infection model of CD4+ T-cells Comput. Biol. Chem. 67, 205-212 (2017)

53. Yüzbași, Ş., Sezer, M.: An exponential approximation for solutions of generalized pantograph-delay differential equations. Appl. Math. Model. 37, 9160-9173 (2013) 
54. Yüzbaşi, Ş., Sezer, M.: Exponential collocation method for solutions of singularly perturbed delay differential equations. Abstr. Appl. Anal. 2013, Article ID 493204 (2013)

55. Yüzbaşi, Ş., Sezer, M.: An exponential matrix method for solving systems of linear differential equations. Math. Methods Appl. Sci. 37, 336-348 (2013)

56. Yüzbaşi, Ş., Sezer, M.: An exponential approach for the system of nonlinear delay integro-differential equations describing biological species living together. Neural Comput. Appl. 27, 769-779 (2016)

Submit your manuscript to a SpringerOpen ${ }^{\circ}$ journal and benefit from:

- Convenient online submission

$\checkmark$ Rigorous peer review

Open access: articles freely available online

- High visibility within the field

- Retaining the copyright to your article

Submit your next manuscript at $\boldsymbol{\Delta}$ springeropen.com 